\title{
Reversible Data Hiding Considering Shifting Direction and Human Visual Sensitivity
}

\author{
Han-Yan $\mathrm{Wu}^{1, \text { a }}$, Yanwei $\mathrm{Li}^{2, \mathrm{~b}}$, Jeanne Chen ${ }^{3, \mathrm{c}}$ and Wien Hong ${ }^{1, \mathrm{~d}}$ \\ ${ }^{1}$ Department of Information Management, Yu Da University, Miaoli, Taiwan \\ ${ }^{2}$ Department of Information Engineering, Zhengzhou Chenggong University of Finance and \\ Economics, China \\ ${ }^{3}$ Department of Computer Science and Information Engineering, National Taichung University of \\ Science and Technology, Taichung, Taiwan \\ a hanyan.wu0414@gmail.com, ${ }^{\mathrm{b}}$ liyanweicc@gmail.com, c jeanne@ntit.edu.tw, \\ dwienhong@ydu.edu.tw
}

Keywords: Embedding level, location map, just noticeable difference.

\begin{abstract}
In view of the technique "A New Histogram Modification Based Reversible Data Hiding Algorithm Considering the Human Visual System" by Jung et al., some modifications have been made on the parts of the causal window and the pixel overflow/underflow in present study. In Jung et al.'s method, the number of reference pixel was obtained based on a causal window so that the prediction value, the just noticeable difference (JND) and others can be determined. However, the causal window used in Jung et al.'s method may reduce the prediction accuracy. In this paper, the modifications on two parts are proposed to turn around this situation. First, the causal window is narrowed to decrease the number of reference pixels so that the prediction accuracy can be enhanced. Second, in dealing with possible pixel underflow or overflow, only the pixels that will definitely underflow or overflow, are modified. Using such modifications both the payload and the image quality can be improved. It is shown by experiments that our modifications not only increase payload but also maintain image quality effectively.
\end{abstract}

\section{Introduction}

Data hiding is a technique to transmit secret information privately by embedding data in carriers that could be audios, videos or images. The most suitable carrier is image since it is often seen and used on network transmission.

There are two types of data hiding methods. In one type the information can be obtained from the stego image at the receiver, but no way to restore it to its original state. This is known as non-reversible $[1,2]$. In the other type the stego image obtained at the receiver can be restored to its original state, which is called as reversible. In recent years many studies on reversible data hiding have been reported while the application of the reversible technique into the encrypted image is also published [3].

Embedding data using difference expansion can be achieved by grouping two adjacent pixels in one, as shown by Tian [4]. But a significant distortion on image by difference expansion leads to an unsatisfactory image quality. $\mathrm{Ni}$ et al. [5] proposed to embed data by shifting pixels between histogram peak and zero. In this way a good image quality can be obtained but the payload is limited by the peak height of image histogram. Thodi and Rodriguez [6] integrate two techniques (histogram-shifting and difference expansion) where embedding data is achieved by using expansion of prediction error and has better performance than prior works.

Tai et al. [7] suggest a reversible technique based on histogram-shifting where the data hiding is carried out by using the histogram of the absolute values of pixel difference. The maximum payload is determined by the embedding level.

In consideration of human visual system (HVS), Jung et al. [8] suggest a reversible technique where a framework is made of the Tai et al. technique. In this technique a casual window is given. The width of the casual window is controlled by parameter $B$. The prediction value and the just 
noticeable difference (JND) are calculated based on the given casual window. The embedding level of each pixel is thus adjusted by JND.

However, in the technique by Jung et al., too many reference pixels are used to calculate the prediction value, resulted in a decreased in prediction accuracy. Therefore, regarding the prediction method by Jung et al, some modifications are proposed in present study to raise the prediction accuracy. Besides, an improved technique is also suggested for the possible underflow or overflow pixels to reduce any unnecessary corrections, so that the size of the location map can be reduced, and the image quality can be maintained.

\section{Related work}

Tai et al.'s method [7] is used for the basic framework for Jung et al.'s [8] technique. Assuming that $I_{r, c}$ and $I e_{r, c}$ are the pixels before and after embedding respectively, where the subscripts indicate the locations of pixels. The prediction value of $I_{r, c}$ is calculated according to Eq. (1).

$$
p_{r, c}=\frac{1}{C\left(\Omega_{r, c}\right)} \sum_{(r, c) \in \Omega_{r, c}} I_{r, c}
$$

where $\Omega_{r, c}$ represents the set of locations of the reference pixels surrounding $I_{r, c}$ within the casual window for $I_{r, c}$ while $C\left(\Omega_{r, c}\right)$ the number of pixels within $\Omega_{r, c}$. Assuming that $B=5$ as shown in Fig. 1, $\Omega_{r, c}$ has 12 locations in total, and also the average value of these locations for 12 pixels is the prediction value $p_{r, c}$ of $I_{r, c}$. Jung et al. use Eq. (2) to determine whether $I_{r, c}$ is located at edges

$$
E_{r, c}= \begin{cases}1, & \text { if } \operatorname{var}\left(\Omega_{r, c}\right)>T_{e}, \\ 0, & \text { otherwise }\end{cases}
$$

where $E_{r, c}$ represents whether $I_{r, c}$ is located at edge, $\operatorname{var}\left(\Omega_{r, c}\right)$ variance of $\Omega_{r, c}, T_{e}$ threshold of edge. The calculation of JND is shown in Eq. (3).

$$
j n d_{r, c}=T_{l}^{r, c}+\lambda\left(T_{a}^{r, c} / T_{l}^{r, c}\right)
$$

In Eq. (3), $\lambda=0.5$ [10], and $T_{l}^{r, c}$ and $T_{a}^{r, c}$ represent luminance adaptation and activity masking of $I_{r, c}$ respectively. $T_{l}^{r, c}$ is determined based on $\Omega_{r, c}$, as shown in Fig. 2. When $I_{r, c}$ is non-edge (e.g. $E_{r, c}=0$ ), it is assumed that $a=10, b=20$, and $c=24$; when $I_{r, c}$ is edge (e.g. $E_{r, c}=1$ ), it is assumed that $a=8, b=18$, and $c=22 . T_{a}^{r, c}$ is defined as the maximum pixel difference value in reference pixels.

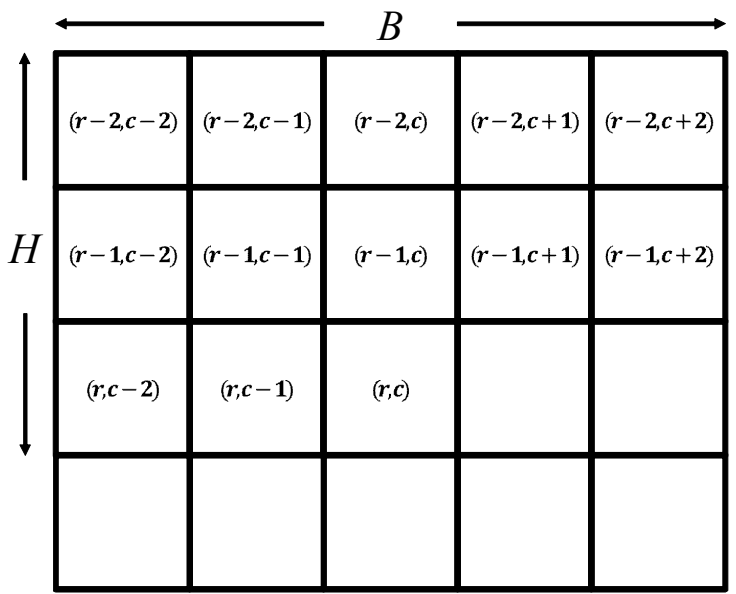

Fig. 1. Causal window

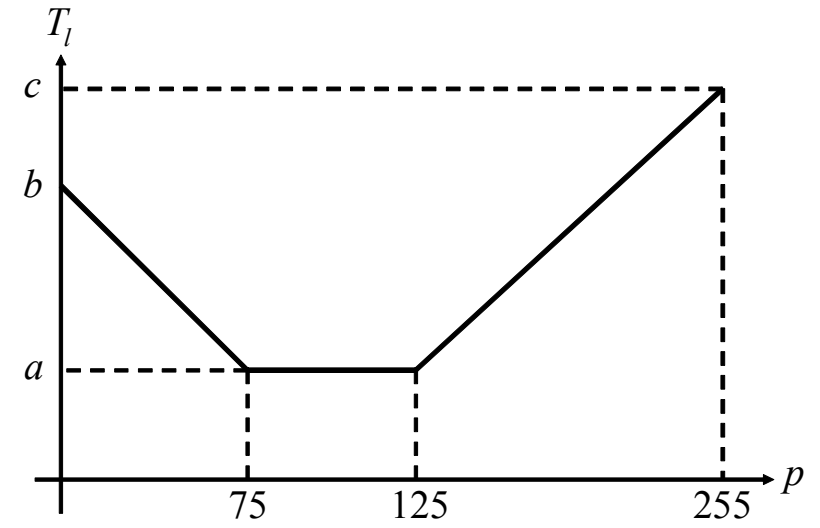

Fig.2. Visibility threshold against background luminance [10] 
After calculating JND the embedding level $K_{r, c}$ is calculated based on Eq. (4) and Eq. (5). When $E_{r, c}=0$, Eq. (4) is used. When $E_{r, c}=1$, Eq. (5) is applied. In Eq. (4) and Eq. (5), $L$ represents maximum embedding level.

$$
\begin{aligned}
& K_{r, c}=\arg _{k} \max 2^{k}, \text { subject to } 2^{k}<j n d_{r, c}, k \leq L . \\
& K_{r, c}=\arg _{k} \min 2^{k}, \text { subject to } 2^{k}>j n d_{r, c}, k \leq L .
\end{aligned}
$$

The detailed procedure of embedding by Jung et al.'s technique is shown as follows.

Input: Cover image $I c$ of size $N \times M$, secret data $S$, maximum embedding level $L$, causal window size $B$.

Output: Stego image Ie.

Step 1. To avoid overflow or underflow after embedding the pixels at both ends of the histogram of $I c$ have to be shifted toward inner. $I c$ is modified based on Eq. (6), resulted in $I$ as the modified value for $I c$.

$$
I_{r, c}= \begin{cases}I c_{r, c}-2^{L}, & I c_{r, c} \geq 255-2^{L}, \\ I c_{r, c}+2^{L}, & I c_{r, c} \leq 2^{L}, \\ I c_{r, c}, & \text { otherwise. }\end{cases}
$$

In Eq. (6), $(r, c)$ represents the location of pixel, $1 \leq r \leq N, 1 \leq c \leq M$. Meanwhile the modified and unmodified pixels in location map $L M$ are indicated by 0 and $1 . L M$ is compressed into $L M^{\prime}$ by JBIG-2 coder [9]. The $L M^{\prime}$ is then concatenated with $S$ to form $S^{\prime}$.

Step 2. Scanning $I$ by using raster-scan order. Let $I_{r, c}$ be the scanned pixel, $H \leq r \leq N$, $1+\lfloor B / 2\rfloor \leq c \leq M-\lfloor B / 2\rfloor$. The prediction value $p_{r, c}$ of $I_{r, c}$ is calculated by Eq. (1). The prediction error $d_{r, c}=\left|I_{r, c}-p_{r, c}\right|$ of $I_{r, c}$ is then calculated. $|x|$ represents the absolute value of $x$.

Step 3. Using Eq. (2) to determine whether $I_{r, c}$ is at edge or non-edge. The $j n d_{r, c}$ of $I_{r, c}$ is then calculated by Eq. (3).

Step 4. Determine the embedding level $K_{r, c}$ of $I_{r, c}$. If $I_{r, c}$ is a non-edge ( $E_{r, c}=0$ ) pixel, Eq. (4) is used; if $I_{r, c}$ is an edge ( $\left.E_{r, c}=1\right)$ pixel, Eq. (5) is applied.

Step 5. If $d_{r, c} \geq 2^{K_{r, c}^{r, c}}$, implying that $I_{r, c}$ is non-embeddable, go to Step 6; if $d_{r, c}<2^{K_{r, c}}$, implying that $I_{r, c}$ is embeddable, $I_{r, c}$ is then modified by Eq. (7) to hide the secret data where the modified value of $I_{r, c}$ is $I e_{r, c}$.

$$
I e_{r, c}= \begin{cases}I_{r, c}+d_{r, c}+b, & I_{r, c} \geq p_{r, c}, \\ I_{r, c}-d_{r, c}-b, & I_{r, c}<p_{r, c} .\end{cases}
$$

Step 6. If $d_{r, c}<2^{K_{r, c}}$, implying that $I_{r, c}$ is non-embeddable, $I_{r, c}$ is modified based on Eq. (8) where the modified value of $I_{r, c}$ is $I e_{r, c}$.

$$
I e_{r, c}= \begin{cases}I_{r, c}+2^{K_{r, c}}, & I_{r, c} \geq p_{r, c}, \\ I_{r, c}-2^{K_{r, c}}, & I_{r, c}<p_{r, c} .\end{cases}
$$

Step 7. Repeat the Steps 2-6 until $S^{\prime}$ is embedded completely.

In order to obtain information from $I e$ and also to restore $I c$ into original state, the lengths of $L M^{\prime}$ and $S^{\prime}$, the maximum embedding level as well as the size of causal window $B$ have to be known at the receiver. The detailed procedure for extracting data and restoring the cover image can be seen in [8]. 


\section{Proposed method}

The technique by Tai et al. [7] is used by Jung et al. [8] as framework, in consideration of human visual system (HVS). In Jung et al.'s technique the number of reference pixels is determined by the parameter $B$ and causal window. Assuming that $B=5,12$ reference pixels are used, as shown in Fig. 1. Because a large causal window is used in Jung et al.'s technique, the pixel correlation may decreased and subsequently reduce the payload. Therefore, in present study the height of the casual window is reduced by deleting the pixels at the top row of Fig. 1 in order to higher the prediction accuracy, hence to enhance the payload.

Basides, in order to avoid any underflow or overflow occuring after embedding, all the pixels that may underflow (pixle values smaller than or equal to $2^{L}$ ) or overflow (pixle values greater than or equal to $255-2^{L}$ ) have to be modified. In reality, an underflow occurs when the pixel is small or equal to $2^{L}$ and also smaller than the prediction value. An overflow occurs when the pixel is greater than or equal to $255-2^{L}$ and also greater than or equal to prediction value. In present study only the pixels that will definitely underflow or overflow, are modified.

The detailed procedure of embedding of the proposed method is shown as follows.

Input: Cover image $I c$ of size $N \times M$, secret data $S$, maximum embedding level $L$, causal window size $B$.

Output: Stego image Ie.

Step 1. Scan $I c$ by using raster-scan order. Let $I c_{r, c}$ be the scanned pixel, $H \leq r \leq N$, $1+\lfloor B / 2\rfloor \leq c \leq M-\lfloor B / 2\rfloor$. The prediction value $p_{r, c}$ of $I c_{r, c}$ is calculated by Eq. (1).

Step 2. Use Eq. (2) to determine whether $I c_{r, c}$ is at edge or non-edge. The $j n d_{r, c}$ of $I c_{r, c}$ is then calculated by Eq. (3).

Step 3. Determine the embedding level $K_{r, c}$ of $I c_{r, c}$. If $I c_{r, c}$ is non-edge ( $\left.E_{r, c}=0\right)$, Eq. (4) is used; if $I c_{r, c}$ is edge $\left(E_{r, c}=1\right)$, Eq. (5) is used.

Step 4. Use Eq. (9) to modify $I c_{r, c}$ in order to avoid any overflow or underflow after embedding. A location map $L M$ is used to record the positions of modified pixels.

$$
I_{r, c}= \begin{cases}I c_{r, c}-2^{K_{r, c}}, & \text { if } I c_{r, c} \geq 255-2^{K_{r, c}} \text { and } I c_{r, c} \geq p_{r, c}, \\ I c_{r, c}+2^{K_{r, c}}, & \text { if } I c_{r, c} \leq 2^{K_{r, c}} \text { and } I c_{r, c}<p_{r, c} \\ I c_{r, c}, & \text { otherwise. }\end{cases}
$$

Step 5. Repeat Steps 1-4 until $r=N, c=\lfloor B / 2\rfloor$.

Step 6. Compress $L M$ into $L M^{\prime}$ by JBIG-2 [9], and the $L M^{\prime}$ is then concatenated with $S$ to form $S^{\prime}$.

Step 7. Scan $I$ using the same order as in Step 1. The prediction error $d_{r, c}=\left|I_{r, c}-p_{r, c}\right|$ of $I_{r, c}$ is calculated.

Step 8. If $d_{r, c} \geq 2^{K_{r, c}}$, implying that $I_{r, c}$ is non-embeddable, go to Step 9; if $d_{r, c}<2^{K_{r, c}}$, implying that $I_{r, c}$ is embeddable, $I_{r, c}$ is then modified by Eq. (7) to hide secret data where the modified value of $I_{r, c}$ is $I e_{r, c}$.

Step 9. If $d_{r, c}<2^{K_{r, c}}$, implying that $I_{r, c}$ is non-embeddable, $I_{r, c}$ is modified based on Eq. (8) where the modified value of $I_{r, c}$ is $I e_{r, c}$.

Step 10. Repeat the Steps 7-9 until $S^{\prime}$ is embedded completely.

Since the extracting secret and restoring image of the proposed method is identical to that of Jung et al.'s method, the procedure concerned will not be restated. 


\section{Experimental result}

As mentioned before, in present study some modifications are made on two aspects to improve the technique by Jung et al. Comparison is thus made on the payload and the image quality among the technique modified and that by Jung et al. Four gray images (Lena, Baboon, Aerial, Sailboat) obtained from the USC-SIPI image database [11] are used for experiment, as shown in Fig. 3. The image quality is measured by peak-to-noise ratio (PSNR) while the embedded data is produced by a pseudo random number generator (PRNG).

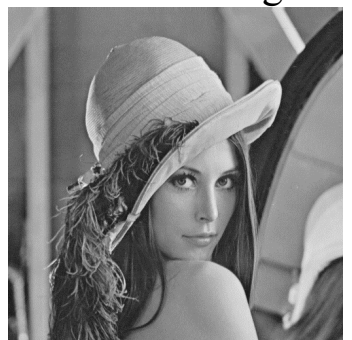

(a) Lena(b) Baboon

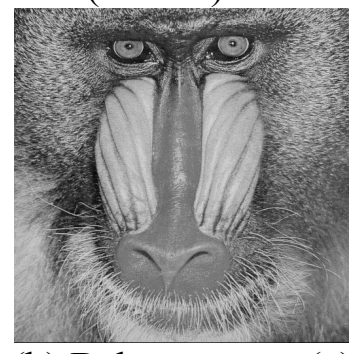

(c) Aerial

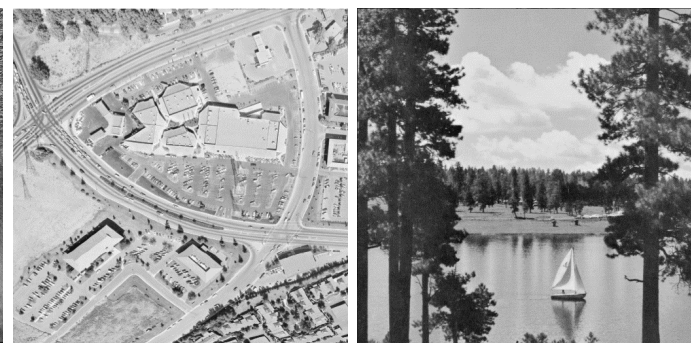

(d) Sailboat

Fig. 3. Four test images.

At first comparison is made on the maximum payload and the image quality between the modified technique and that by Jung et al. as shown in Table 1 where $B=3$ and $T_{e}=200$, and the embedding level $L$ varies from 0 to 4 . It is seen from Table 1 that for the same embedding levels the payload of the proposed method is larger than that by Jung et al.'s method. This means that the proposed method offers a higher payload.

Table 1. Comparing the maximum payload between the proposed method and Jung et al.'s method

\begin{tabular}{|c|r|r|r|r|r|r|r|r|r|r|}
\hline \multirow{2}{*}{$\begin{array}{c}\text { Cover } \\
\text { image }\end{array}$} & \multicolumn{2}{|c|}{$L=0$} & \multicolumn{2}{c|}{$L=1$} & \multicolumn{2}{c|}{$L=2$} & \multicolumn{2}{|c|}{$L=3$} & \multicolumn{2}{c|}{$L=4$} \\
\cline { 2 - 12 } & Jung et al. & Proposed & Jung et al. & Proposed & Jung et al. & Proposed & Jung et al. & Proposed & Jung et al. & Proposed \\
\hline Lena & 27506 & 28964 & 78749 & 82428 & 151934 & 158457 & 210496 & 218357 & 237905 & 243475 \\
\hline baboon & 8135 & 8972 & 24217 & 26285 & 54381 & 59571 & 103346 & 111674 & 160483 & 168831 \\
\hline Aerial & 17475 & 22058 & 49454 & 60370 & 93557 & 110193 & 141221 & 160206 & 189286 & 208109 \\
\hline Sailboat & 18247 & 19743 & 52117 & 56647 & 105266 & 112961 & 167292 & 177618 & 206826 & 216390 \\
\hline Avg. bits & 17705 & 19934 & 51134 & 56433 & 101285 & 110296 & 155589 & 166964 & 198625 & 209201 \\
\hline $\begin{array}{c}\text { Avg. } \\
\text { PSNR }\end{array}$ & 48.32 & 48.33 & 42.64 & 42.69 & 37.45 & 37.58 & 32.86 & 33.16 & 28.97 & 29.57 \\
\hline $\begin{array}{c}\text { Gain in } \\
\text { payload }\end{array}$ & \multicolumn{2}{|c|}{2229} & \multicolumn{2}{|c|}{5298} & \multicolumn{2}{|c|}{9011} & & 11375 & & 10576 \\
\hline
\end{tabular}

A comparison is also made on the lengths of location maps between our technique and that by Jung et al. in Table 2. It is shown that the proposed method may reduce the size of the location maps effectively indeed. In particular, the larger the embedding level is, the more obvious the effectiveness will be. For example, it can be seen in Table 2 that the gain in payload is 1310 bits when the embedding lavel is 4 .

Table 2. Comparing the location map between the proposed method and Jung et al.'s method

\begin{tabular}{|c|r|r|r|r|r|r|r|r|r|r|}
\hline \multirow{2}{*}{$\begin{array}{c}\text { Cover } \\
\text { image }\end{array}$} & \multicolumn{2}{|c|}{$L=0$} & \multicolumn{2}{c|}{$L=1$} & \multicolumn{2}{c|}{$L=2$} & \multicolumn{2}{|c|}{$L=3$} & \multicolumn{2}{|c|}{$L=4$} \\
\cline { 2 - 11 } & Jung et al. & Proposed & Jung et al. & Proposed & Jung et al. & Proposed & Jung et al. & Proposed & Jung et al. & Proposed \\
\hline Lena & 312 & 312 & 312 & 312 & 312 & 312 & 312 & 312 & 360 & 360 \\
\hline baboon & 440 & 440 & 584 & 584 & 664 & 664 & 632 & 656 & 2240 & 1320 \\
\hline Aerial & 2168 & 312 & 2168 & 312 & 3024 & 1168 & 5464 & 3648 & 10920 & 8896 \\
\hline Sailboat & 312 & 312 & 416 & 312 & 560 & 312 & 720 & 312 & 2800 & 504 \\
\hline Avg. bits & 808 & 344 & 870 & 380 & 1140 & 614 & 1782 & 1232 & 4080 & 2770 \\
\hline $\begin{array}{c}\text { Gain in } \\
\text { payload }\end{array}$ & \multicolumn{3}{|c|}{464} & \multicolumn{2}{|c|}{490} & \multicolumn{2}{|c|}{526} & \multicolumn{2}{|c|}{550} & \multicolumn{2}{|c}{1310} \\
\hline
\end{tabular}




\section{Conclusion}

Modifications on two parts are proposed for the technique "A New Histogram Modification Based Reversible Data Hiding Algorithm Considering the Human Visual System" by Jung et al. In the first part, the causal window is narrowed to reduce the number of reference pixels, hence to raise the payload. In the second part, the modification is made only on the pixels that will definitely underflow or overflow. In this way the length of location map is decreased to increase payload. The experiment shows the method that may increase payload and also maintain image quality.

\section{References}

[1] W. Hong and T.S. Chen, A novel data embedding method using adaptive pixel pair matching, IEEE Trans. Inf. Forensics Security 7 (2012) 176-184.

[2] W. Hong, T.S. Chen, and C.W. Luo, Data embedding using pixel value differencing and diamond encoding with multiple-base notational system, Journal of Systems and Software, 85 (2012) 1166-1175.

[3] W. Hong, T.S. Chen, and H.Y. Wu, An improved reversible data hiding in encrypted images using side match, IEEE Signal Process. Lett. 19 (2012) 199-202.

[4] J. Tian, Reversible data embedding using a difference expansion, IEEE Trans. Circuits Syst. Video Technol. 13 (2003) 890-896.

[5] Z. Ni, Y.Q. Shi, N. Ansari, and W. Su, Reversible data hiding, IEEE Trans. Circuits Syst. Video Technol., 16, (2006) 354-362.

[6] D.M. Thodi and J.J. Rodriguez, Expansion embedding techniques for reversible watermarking, IEEE Trans. Image Process. 16 (2007) 721-730.

[7] W.L. Tai, C.M. Yeh, and C.C. Chang, Reversible data hiding based on histogram modification of pixel differences, IEEE Trans. Circuits Syst. Video Technol. 19 (2009) 906-910.

[8] S.W. Jung, L.T. Ha, and S.J. Ko, A new histogram modification based reversible data hiding algorithm considering the human visual system, IEEE Signal Process. Lett. 18 (2011) 95-98.

[9] P. Howard, F. Kossentini, B. Martins, S. Forchhammer, and W. Rucklidge, The emerging JBIG2 standard, IEEE Trans. Circuits Syst. Video Technol. 8 (1998) 838-848.

[10]W. Lin, L. Dong, and P. Xue, Visual distortion gauge based on discrimination of noticeable contrast changes, IEEE Trans. Circuits Syst. Video Technol. 15 (2005) 900-909.

[11]USC-SIPI image database on http://sipi.use.edu/database/. 